\title{
Joint Probability Density Functions for Direct-Detection Optical Receivers
}

\author{
Jae Seung Lee* \\ Department of Electronic Engineering, Kwangwoon University, Kwangwoon-ro 20, Nowon-gu, \\ Seoul 139-701, Korea
}

(Received February 11, 2014 : revised March 21, 2014 : accepted March 21, 2014)

\begin{abstract}
We derive joint probability density functions (JPDFs) for two adjacent data from direct-detection optical receivers in dense wavelength-division multiplexing systems. We show that the decision using two data per bit can increase the receiver sensitivity compared with the conventional decision. Our JPDFs can be used for software-defined optical receivers enhancing the receiver sensitivities for intensity-modulated channels.

Keywords: Optical communication, Optical receivers, Optical amplifiers, Gaussian optical receiver OCIS codes : (060.2330) Fiber optics communications; (060.0060) Fiber optics and optical communications; (060.4510) Optical communications; (060.2340) Fiber optics components
\end{abstract}

\section{INTRODUCTION}

Recently, software-defined networks that can provide practical ways of handling multi-standard environments have been investigated extensively [1, 2]. In a similar way, softwaredefined optical receivers are under development in optical fiber communication systems to satisfy many different kinds of modulation formats and baud rates [3, 4]. Although coherent optical systems have become more practical than during their early stages, currently deployed optical transmission systems are mostly the intensity-modulated and direct-detection (IM/DD) type.

Conventional optical receivers for IM/DD systems use a single data per single bit for the decision [5] and require clock recovery circuits. If we could use multiple data for the decision, we do not need the clock recovery circuits [4] while enhancing the receiver sensitivities. However, there are no analyses, to our knowledge, about the correlations between two adjacent data from an optical receiver.

In this paper, we propose to use correlations of two adjacent data for the decision in direct-detection optical receivers. Using the receiver eigenmodes [6-12], we derive the joint characteristic function (JCF) for two successive data from an optical receiver and evaluate the corresponding joint probability density function (JPDF) [13]. The receiver eigenmodes can describe accurately the effects of the amplified spontaneous emission (ASE), received optical waveforms, and shapes of optical and electrical filters within the receiver
[7]. Recently, receiver eigenmode contributions have been analyzed as a function of time for the optical receiver output [12]. It has been found that, in conventional dense WDM systems [14-17], where the channel spacing is comparable to the bit rate, the lowest-order (0-th) receiver eigenmode contributes dominantly. We will use this fact to find the correlations of two adjacent data and the threshold line for the decision to get higher sensitivities than conventional receivers.

\section{JOINT CHARACTERISTIC FUNCTION}

Before the derivation of the JPDF, we derive the JCF first. We will consider two adjacent samples at times $t_{1}$ and $t_{2}$. Considering only the polarization component parallel to the received optical signal, we can find the voltage at $t_{1}$, denoted as $y_{1}$, as [7]

$$
y_{1}=\frac{k}{8 \pi^{2}} \sum_{m} \frac{\left|S_{m}\left(t_{1}\right)+N_{m}\left(t_{1}\right)\right|^{2}}{\lambda_{m}},
$$

where $S_{m}\left(t_{1}\right)$ and $N_{m}\left(t_{1}\right)$ are complex numbers that represent the signal and the noise amplitudes, respectively, for the $m$-th receiver eigenmode. $\lambda_{m}$ is the $m$-th eigenvalue and $k$ is a proportional constant. For all $m$, the real and the imaginary parts of $N_{m}\left(t_{1}\right)$ are mutually independent zero

\footnotetext{
*Corresponding author: jslee@kw.ac.kr
} 
mean Gaussian random variables having an identical variance of $\sigma_{\omega}^{2}$. In conventional dense WDM systems, the lowestorder receiver eigenmode contributes dominantly [12] and we can approximate

$$
y_{1}=\frac{k}{8 \pi^{2}} \frac{\left|S_{0}\left(t_{1}\right)+N_{0}\left(t_{1}\right)\right|^{2}}{\lambda_{0}} .
$$

We normalize $y_{1}$ such that it becomes a power ratio such that

$$
\bar{y}_{1}=\frac{\left|S_{0}\left(t_{1}\right)+N_{0}\left(t_{1}\right)\right|^{2}}{2 \sigma_{\omega}^{2}},
$$

where the denominator is the noise power per receiver eigenmode per polarization. Similarly, we have the normalized voltage at $t_{2}$ as

$$
\bar{y}_{2}=\frac{\left|S_{0}\left(t_{2}\right)+N_{0}\left(t_{2}\right)\right|^{2}}{2 \sigma_{\omega}^{2}}
$$

$N_{0}\left(t_{1}\right)$ and $N_{0}\left(t_{2}\right)$ are related by a correlation function as [12]

$$
N_{0}\left(t_{1}\right)=C\left(t_{1}-t_{2}\right) N_{0}\left(t_{2}\right) .
$$

The correlation function, $C\left(t_{1}-t_{2}\right)$, relates the 0 -th order eigenmode amplitudes at different times, which is given by

$$
C\left(t_{1}-t_{2}\right)=\int d \omega \phi_{0}(\omega) \phi_{0}^{*}(\omega) \exp \left\{j \omega\left(t_{1}-t_{2}\right)\right\},
$$

where $\phi_{0}(\omega)$ is the 0 -th order eigenfunction. Let's denote the real part of $N_{0}\left(t_{1}\right)$ and $N_{0}\left(t_{2}\right)$ as $N_{1 r}$ and $N_{2 r}$, respectively, and the imaginary part of $N_{0}\left(t_{1}\right)$ and $N_{0}\left(t_{2}\right)$ as $N_{1 i}$ and $N_{2 i}$, respectively. The covariance matrix for $N_{1 r}, N_{1 i}, N_{2 r}$, and $N_{2 i}$ can be found as [13]

$$
\Sigma=\sigma_{\omega}^{2}\left(\begin{array}{cccc}
1 & 0 & C_{r} & -C_{i} \\
0 & 1 & C_{i} & C_{r} \\
C_{r} & C_{i} & 1 & 0 \\
-C_{i} & C_{r} & 0 & 1
\end{array}\right)
$$

where $C_{r}$ and $C_{i}$ are real and imaginary parts of $C\left(t_{1}-t_{2}\right)$, respectively. The inverse matrix of $\Sigma$ is

$$
\Sigma^{-1}=\frac{1}{G \sigma_{\omega}^{2}}\left(\begin{array}{cccc}
1 & 0 & -C_{r} & C_{i} \\
0 & 1 & -C_{i} & -C_{r} \\
-C_{r} & -C_{i} & 1 & 0 \\
C_{i} & -C_{r} & 0 & 1
\end{array}\right)
$$

where $G=1-C_{r}^{2}-C_{i}^{2}$.

For the Gaussian vector, $X=\left(N_{1 r}, N_{1 i}, N_{2 r}, N_{2 i}\right)^{T}$, where $T$ means the transpose, its JPDF is [13]

$$
f_{X}(x)=\frac{1}{(2 \pi)^{2}|\Sigma|^{1 / 2}} \exp \left(-\frac{1}{2} x^{T} \Sigma^{-1} x\right) .
$$

Thus the JPDF for $N_{1 r}, N_{1 i}, N_{2 r}$, and $N_{2 i}$ can be written as

$$
\begin{aligned}
& f_{X}\left(N_{1 r}, N_{1 i}, N_{2 r}, N_{2 i}\right)=\frac{1}{4 \pi^{2} G \sigma_{\omega}^{4}} \\
& \cdot \exp \left[-\frac{\left\{\left(N_{1 r}^{2}+N_{1 i}^{2}+N_{2 r}^{2}+N_{2 i}^{2}\right) / 2-C_{r} N_{1 r} N_{2 r}-C_{r} N_{1 i} N_{2 i}+C_{i} N_{1 r} N_{2 i}-C_{i} N_{1 i} N_{2 r}\right\}}{G \sigma_{\omega}^{2}}\right] .
\end{aligned}
$$

The JCF for $\bar{y}_{1}$ and $\bar{y}_{2}$ can be written as

$$
\begin{aligned}
\Phi\left(\xi_{1}, \xi_{2}\right) & =E\left\{\exp \left(j \xi_{1} \bar{y}_{1}+j \xi_{2} \bar{y}_{2}\right)\right\} \\
& =\int d N_{1 r} d N_{1 i} d N_{2 r} d N_{2 i} \exp \left(j \xi_{1} \bar{y}_{1}+j \xi_{2} \bar{y}_{2}\right)
\end{aligned}
$$

where $E\{\cdot\}$ is the ensemble average. The integrations can be done exactly using the Gaussian integration formula,

$$
\int_{-\infty}^{\infty} d x \exp \left(-\alpha x^{2}-2 \beta x\right)=\sqrt{\frac{\pi}{\alpha}} \exp \left(\frac{\beta^{2}}{\alpha}\right)
$$

where $\alpha$ and $\beta$ are constants with $\operatorname{Re} \alpha>0$. Thus we find

$$
\Phi\left(\xi_{1}, \xi_{2}\right)=\frac{\exp \left(\frac{j \xi_{1} A_{1}+j \xi_{2} A_{2}+\xi_{1} \xi_{2} B}{1-j \xi_{1}-j \xi_{2}-G \xi_{1} \xi_{2}}\right)}{\left(1-j \xi_{1}-j \xi_{1}-G \xi_{1} \xi_{2}\right)}
$$

where

$$
\begin{aligned}
& A_{1}=\left|S_{0}\left(t_{1}\right)\right|^{2} / 2 \sigma_{\omega}^{2}, \\
& A_{2}=\left|S_{0}\left(t_{2}\right)\right|^{2} / 2 \sigma_{\omega}^{2}, \\
& B=A_{1}+A_{2}-2 H, \\
& H=\left\{C_{r}\left(S_{1 r} S_{2 r}+S_{1 i} S_{2 i}\right)+C_{i}\left(S_{1 i} S_{2 r}-S_{1 r} S_{2 i}\right)\right\} / 2 \sigma_{\omega}^{2} .
\end{aligned}
$$

$S_{1 r}$ and $S_{1 i}$ are real and imaginary parts of $S_{0}\left(t_{1}\right)$, respectively. Also, $S_{2 r}$ and $S_{2 i}$ are real and imaginary parts of $S_{0}\left(t_{2}\right)$, respectively. If we include the other polarization, where only the ASE components are present, we obtain the JCF exactly as follows: 


$$
\Phi\left(\xi_{1}, \xi_{2}\right)=\frac{\exp \left(\frac{j \xi_{1} A_{1}+j \xi_{2} A_{2}+\xi_{1} \xi_{2} B}{1-j \xi_{1}-j \xi_{2}-G \xi_{1} \xi_{2}}\right)}{\left(1-j \xi_{1}-j \xi_{1}-G \xi_{1} \xi_{2}\right)^{2}}
$$

\section{JOINT PROBABILITY DENSITY FUNCTION}

The JPDF of $\bar{y}_{1}$ and $\bar{y}_{2}$ can be found from the twodimensional Fourier transform

$$
f\left(\bar{y}_{1}, \bar{y}_{2}\right)=\frac{1}{(2 \pi)^{2}} \int d \xi_{1} d \xi_{2} \Phi\left(-\xi_{1},-\xi_{2}\right) \exp \left(j \xi_{1} \bar{y}_{1}+j \xi_{2} \bar{y}_{2}\right) .
$$

When the signal is absent, the JCF becomes

$$
\Phi\left(\xi_{1}, \xi_{2}\right)=\frac{1}{\left(1-j \xi_{1}-j \xi_{2}-G \xi_{1} \xi_{2}\right)^{2}},
$$

which gives the JPDF of $\bar{y}_{1}$ and $\bar{y}_{2}$ in an exact form

$$
\begin{aligned}
f\left(\bar{y}_{1}, \bar{y}_{2}\right)= & \frac{1}{G} \sqrt{\frac{\bar{y}_{1} \bar{y}_{2}}{1-G}} \exp \left\{-\left(\bar{y}_{1}+\bar{y}_{2}\right) / G\right\} \\
& \cdot I_{1}\left(\frac{2}{G} \sqrt{\bar{y}_{1} \bar{y}_{2}(1-G)}\right),
\end{aligned}
$$

where $I_{1}$ is the modified Bessel function. We have used the following relation $(x, M>0)$ :

$$
\begin{aligned}
& \frac{1}{2 \pi} \int_{-\infty}^{\infty} d \xi \frac{\exp \left(\frac{-j \xi M}{1+j \xi}\right)}{(1+j \xi)^{2}} \exp (-j \xi x) \\
& =\sqrt{\frac{x}{M}} \exp (-M-x) I_{1}(2 \sqrt{x M})
\end{aligned}
$$

If the two data are independent, we find from (22)

$$
f\left(\bar{y}_{1}, \bar{y}_{2}\right)=\bar{y}_{1} \bar{y}_{2} \exp \left\{-\left(\bar{y}_{1}+\bar{y}_{2}\right)\right\}
$$

This JPDF is just a product of each sample's PDF. To find (24), we have increased $G$ satisfying the $G<1$ condition. When the signal is present, the JPDF can be obtained numerically. Note that the integration over $\xi_{1}$ can be done exactly to yield

$$
\begin{aligned}
f\left(\bar{y}_{1}, \bar{y}_{2}\right)= & \frac{\bar{y}_{1}}{\pi} \exp \left\{-\left(\bar{y}_{1}+B\right) / G\right\} \\
& \cdot \int d \xi_{2} \frac{\exp \left(j \xi_{2} \bar{y}_{2}\right) I_{1}(W)}{\left(1+j G \xi_{2}\right)^{2}} \\
& \cdot \exp \left\{-\frac{A_{1}-B / G+\bar{y}_{1}(1-1 / G)}{1+j G \xi_{2}}\right\},
\end{aligned}
$$

where

$$
W=2 \sqrt{\bar{y}_{1}\left(\frac{A_{1}+j \xi_{2} B}{1+j G \xi_{2}}-\frac{j \xi_{2} A_{2}}{1+j \xi_{2}}\right)\left(\frac{1+j \xi_{2}}{1+j G \xi_{2}}\right)} .
$$

The remaining integral can be done using a fast-Fouriertransform algorithm.

We may use the asymptotic form for the Bessel function, $I_{1}(x) \sim \exp (x) / \sqrt{2 \pi x}(x>>1)$, and evaluate the integral of (25) using the method of steepest descents [18], which gives

$$
\begin{aligned}
& f\left(\bar{y}_{1}, \bar{y}_{2}\right)=\frac{\left(\bar{y}_{1} \bar{y}_{2}\right)^{1 / 4}}{4 \pi\left(A_{1} A_{2}\right)^{3 / 4}} \\
& \cdot \exp \left[-\left(\sqrt{\bar{y}_{1}}-\sqrt{A_{1}}\right)^{2}\left\{1+\frac{H^{2}}{A_{1} A_{2}} \sqrt{\frac{\bar{y}_{2}}{A_{2}}}+(1-G)\left(1-\sqrt{\frac{\bar{y}_{2}}{A_{2}}}\right)\right\}\right. \\
& -\left(\sqrt{\bar{y}_{2}}-\sqrt{A_{2}}\right)^{2}\left\{1+\frac{H^{2}}{A_{1} A_{2}} \sqrt{\frac{\bar{y}_{1}}{A_{1}}}+(1-G)\left(1-\sqrt{\frac{\bar{y}_{1}}{A_{1}}}\right)\right\} \\
& \left.-2 H\left(1-\sqrt{\frac{\bar{y}_{1}}{A_{1}}}\right)\left(1-\sqrt{\frac{\bar{y}_{2}}{A_{2}}}\right)\right] \\
& +\left[1+\frac{3 H}{2}\left(\frac{1}{A_{1}}+\frac{1}{A_{2}}-\frac{\sqrt{\bar{y}_{1}}}{\sqrt{A_{1}} A_{2}}-\frac{\sqrt{\bar{y}_{2}}}{A_{1} \sqrt{A_{2}}}\right)\right. \\
& +H^{2}\left\{\frac{21}{8}\left(\frac{1}{A_{1}^{2}}+\frac{1}{A_{2}^{2}}+\frac{\bar{y}_{1}}{A_{1} A_{2}^{2}}+\frac{\bar{y}_{2}}{A_{1}^{2} A_{2}}\right)+\frac{7}{2} \frac{\sqrt{\bar{y}_{1} \bar{y}_{2}}}{A_{1}^{3 / 2} A_{2}^{3 / 2}}\left(\sqrt{\frac{\bar{y}_{1}}{A_{1}}}+\sqrt{\frac{\bar{y}_{2}}{A_{2}}}\right)-\frac{21}{4}\left(\frac{1}{A_{2}^{2}} \sqrt{\frac{\bar{y}_{1}}{A_{1}}}+\frac{1}{A_{1}^{2}} \sqrt{\frac{\bar{y}_{2}}{A_{2}}}\right)\right\} \\
& +(1-G)\left\{-\frac{3}{4}\left(\frac{A_{1}}{A_{2}}+\frac{A_{2}}{A_{1}}+\frac{\bar{y}_{1}}{A_{2}}+\frac{\bar{y}_{2}}{A_{1}}\right)\right. \\
& \left.\left.+\frac{3}{2}\left(\frac{A_{1}+A_{2}}{A_{1} A_{2}}\right)\left(\sqrt{A_{1} \bar{y}_{1}}+\sqrt{A_{2} \bar{y}_{2}}\right)-2 \sqrt{\frac{\bar{y}_{1}}{A_{1}}} \frac{\bar{y}_{2}}{A_{2}}-2\right\}\right] .
\end{aligned}
$$

The complicated expression of (27) is valid when the correlation is low $(G \approx 1)$. We perform numerical evaluations of (25) to find BER throughout.

\section{RESULTS AND DISCUSSION}

For our analysis, we use a Gaussian optical receiver [11, 12], where both optical and electrical filters are Gaussian. We assume $A_{1}=A_{2}$. We choose the $3-\mathrm{dB}$ bandwidth of the optical filter two times larger that of the electrical filter. The $3-\mathrm{dB}$ bandwidth of the electrical filter is 0.7 times the bit rate. Then the correlation function (6) becomes $C(\tau)=\exp \left[-(0.7 \pi)^{2} 2 q /\left\{\left(1-q^{2}\right) \ln 2\right\}(\tau / T)^{2}\right]$ [12]. We have $q=0.268$ according to the bandwidth ratio between the optical and the electrical filters. $T$ is the bit period. The two sampling points are $T / 4$ apart and we obtain $G=0.396$. We assume no phase changes of the 
signal between two sampling points, which gives $H=$ $A_{1} \sqrt{1-G}$.

Figure 1 shows two JPDFs evaluated numerically along the $\bar{y}_{1}=\bar{y}_{2}$ line for $A_{1}=40.65$ and for $A_{1}=0$ simultaneously. The former corresponds to the mark and the latter corresponds to the space. The JPDF for the space has been scaled down by the factor of 10 compared with the JPDF for the mark.

In Fig. 2, we show the foregoing JPDFs in a 3dimensional way. It has been obtained by adding the JPDFs' functional values to $\bar{y}_{2}$ after multiplying a constant $4 \times 10^{3}$. Only the points, where at least one of the JPDFs is larger than $10^{-10}$, are shown. The JPDF for the space has been scaled down by the factor of 10 here also. Since $A_{1}$ $=A_{2}$, the JPDFs, including (22), are symmetric with respect to the $\bar{y}_{1}=\bar{y}_{2}$ line. Note that $f\left(\bar{y}_{1}, \bar{y}_{2}\right)=0$ when

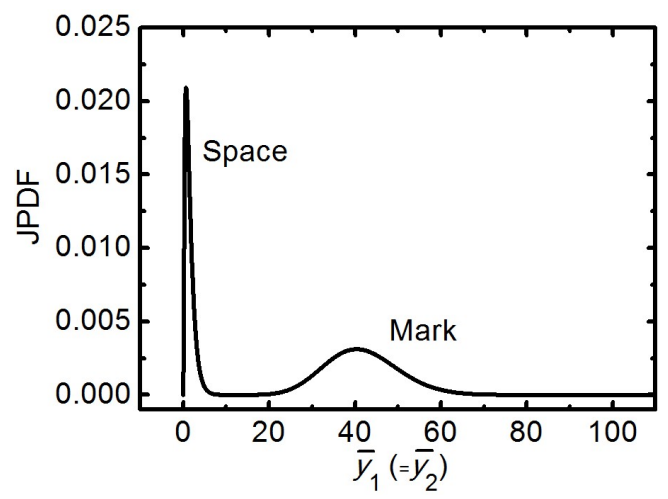

FIG. 1. Joint probability density functions (JPDFs) evaluated numerically along the $\bar{y}_{1}=\bar{y}_{2}$ line for the mark $\left(A_{1,2}=40.65\right)$ and for the space $\left(A_{1,2}=0\right)$. The JPDF for the space has been scaled down by the factor of 10 compared with the JPDF for the mark. The two sampling points are $T / 4$ apart, where $T$ is the bit period. A Gaussian optical receiver is used with $G=$ 0.396 .

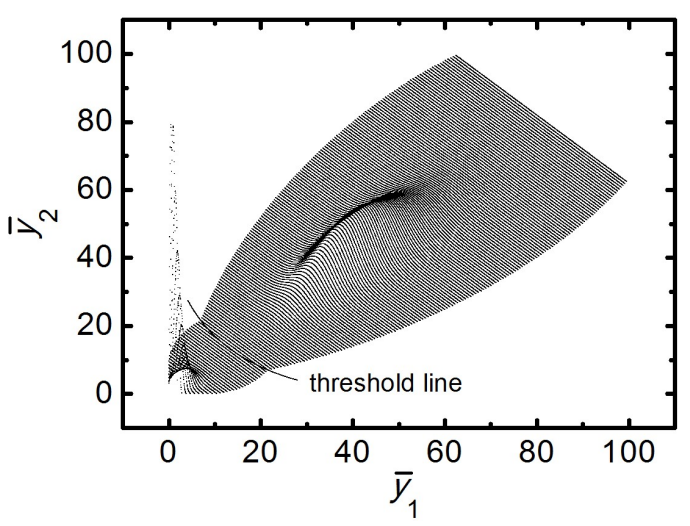

FIG. 2. Plot of the JPDFs of Fig. 1 in the $\left(\bar{y}_{1}, \bar{y}_{2}\right)$ plane. We have added the JPDF's functional value to $\bar{y}_{2}$ after multiplying a constant $4 \times 10^{3}$. We show only the points where at least one of JPDFs is larger than $10^{-10}$. The JPDF for the space has been scaled down by the factor of 10 . at least one of $\bar{y}_{1}$ and $\bar{y}_{2}$ is zero. Along the $\bar{y}_{1}=\bar{y}_{2}$ line, the width of each JPDF increases as $\bar{y}_{1}$ increases. In conventional optical receivers, where a threshold voltage is used, we have $B E R=\left(2 A_{1} / \pi\right)^{1 / 4} \exp \left(-A_{1} / 4\right) / 4$ [11], which is $2.2 \times 10^{-5}$. In our case, there is a threshold line as is shown in Fig. 2 where both JPDFs have the same value. The area of each JPDF beyond that line contributes to the BER [5] which is evaluated numerically as $6.8 \times 10^{-6}$. This corresponds to about $0.5 \mathrm{~dB}$ enhancement in $A_{1}$ compared with the conventional BER of $2.2 \times 10^{-5} . A_{1}$ and $A_{2}$ are actually signal-to-noise ratios [11]. Thus we can have higher system margins using the correlation between the data from the direct-detection optical receiver.

If the two sampling points are $T / 2$ apart with all other parameters fixed, $G$ increases to 0.866 . The JPDFs in this case are shown in Fig. 3, which become more circularly symmetric owing to the reduced correlation. The BER is $2.6 \times 10^{-7}$ and the enhancement in $A_{1}$ increases to about 1.6 $\mathrm{dB}$. If the two sampling points are $3 T / 4$ apart with all other parameters fixed, $G$ increases to 0.989 . The BER is $1.3 \times 10^{-8}$ and the enhancement in $A_{1}$ is about $2.4 \mathrm{~dB}$. If the received signal has a different phase between two sampled points, this will decrease $H$ in (18). In this case, our numerical analyses show that the BER decreases as the phase difference increases. Thus our BER values can be reduced introducing the phase change within a bit.

In order to use the threshold line for the decision, we need analog-to-digital converters (ADCs) and digital signal processing (DSP) circuits instead of conventional D-flip-flop type decision. The speed of ADC and DSP circuits has been increased remarkably up to coherent $200 \mathrm{~Gb} / \mathrm{s}$ per channel during recent years [19]. Realizations of softwaredefined optical receivers using these devices are now technically feasible and our JPDFs can be used to upgrade their capabilities for IM/DD channels.

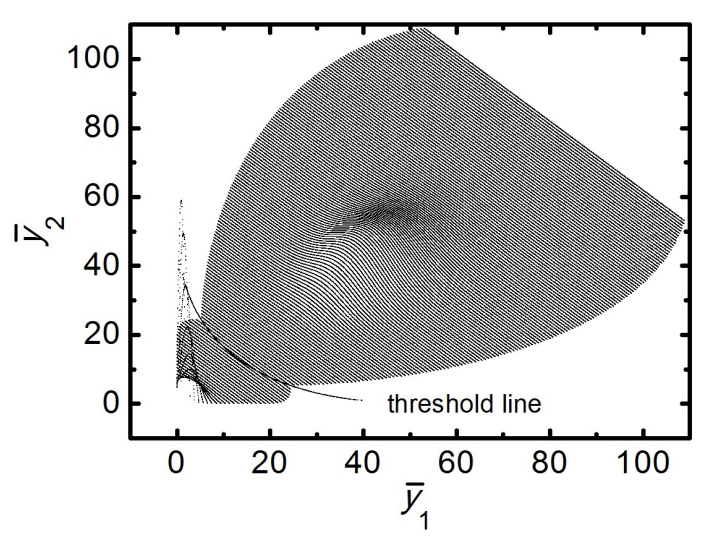

FIG. 3. Plot of the JPDFs in $\left(\bar{y}_{1}, \bar{y}_{2}\right)$ plane, where the two sampling points are $T / 2$ apart with all other parameters the same as Fig. 2. $G=0.866$. The JPDFs become more circularly symmetric than those of Fig. 2 owing to the reduced correlation. 


\section{CONCLUSION}

We have derived the JPDFs for two voltage data from direct-detection optical receivers in dense WDM systems. We have shown that, with our JPDFs, we can reduce the BER and enhance the receiver sensitivities by $0.5 \mathrm{~dB} \sim$ $2.4 \mathrm{~dB}$. Our decision method can be used for software-defined optical receivers supporting both coherent and intensitymodulated channels simultaneously.

\section{ACKNOWLEDGMENT}

The work reported in this paper was conducted during the sabbatical year of Kwangwoon University in 2012 and this research was also supported partly by the Basic Science Research Program through the National Research Foundation of Korea (NRF) funded by the Ministry of Education, Science, and Technology (2013R1A1A2012918).

\section{REFERENCES}

1. M. Channegowda, R. Nejabati, and D. Simeonidou, "Softwaredefined optical networks technology and infrastructure: Enabling software-defined optical network operations [invited]," IEEE/OSA Journal of Optical Communications and Networking 5, A274-A282 (2013).

2. Y. Cui, S. Xiao, C. Liao, I. Stojmenovic, and M. Li, "Data centers as software defined networks: Traffic redundancy elimination with wireless cards at routers," IEEE Selected Areas in Communications 31, 2658-2672 (2013).

3. E. Ip, P. Ji, E. Mateo, Y.-K. Huang, L. Xu, D. Qian, and N. Bai, and T. Wang, " $100 \mathrm{G}$ and beyond transmission technologies for evolving optical networks and relevant physical-layer issues," Proc. IEEE 100, 1065-1078 (2012).

4. P. Isautier, A. Stark, K. Mehta, R. de Salvo, and S. E. Ralph, "Autonomous software-defined coherent optical receivers," in Proc. OFC (Anaheim Convention Center, Anaheim, California, USA, 2013), OTh3B.4-1 OTh3B.4-3.

5. S. D. Personick, "Receiver design for digital fiber optic communications systems, I," Bell Syst. Tech. J. 52, 843874 (1973).

6. M. Kac and A. J. F. Siegert, "On the theory of noise in radio receivers with square law detectors," J. Appl. Phys. 18, 383-397 (1947).

7. J. S. Lee and C. S. Shim, "Bit-error-rate analysis of optically preamplified receivers using an eigenfunction expansion method in optical frequency domain," J. Lightwave Technol. 12, 1224-1229 (1994).

8. E. Forestieri and M. Secondini, "On the error probability evaluation in lightwave systems with optical amplification," J. Lightwave Technol. 27, 706-717 (2009).

9. P. J. Winzer, M. Pfennigbauer, M. M. Strasser, and W. R. Leeb, "Optimum filter bandwidths for optically preamplified NRZ and RZ receivers," J. Lightwave Technol. 19, 12631273 (2001).

10. R. Holzlohner, V. S. Grigoryan, C. R. Menyuk, and W. L. Kath, "Accurate calculation of eye diagrams and bit error rates in optical transmission systems using linearization," J. Lightwave Technol. 20, 389-400 (2002).

11. J. S. Lee and A. E. Willner, "Analysis of Gaussian optical receivers," J. Lightwave Technol. 31, 2987-2993 (2013).

12. K. H. Seo, J. S. Lee, and A. E. Willner. "Time-dependent analysis of optical receivers using receiver eigenmodes," J. Opt. Soc. Korea 17, 305-311 (2013).

13. A. Papoulis and S. U. Pillai, Probability, Random Variables, and Stochastic Processes, 4th ed. (McGraw-Hill, New York, USA, 2002).

14. Y. S. Hurh, G. S. Hwang, J. Y. Jeon, K. G. Lee, K. W. Shin, S. S. Lee, K. Y. Yi, and J. S. Lee, " $1-\mathrm{Tb} / \mathrm{s}(100 \times$ $12.4 \mathrm{~Gb} / \mathrm{s}$ ) transmission of 12.5-GHz-spaced ultradense WDM channels over a standard single-mode fiber of 1200 km," IEEE Photon. Technol. Lett. 17, 696-698 (2005).

15. S. Y. Kim, K. H. Seo, and J. S. Lee, "Spectral efficiencies of channel-interleaved bidirectional and unidirectional ultradense WDM for metro applications," J. Lightwave Technol. 30, 229-233 (2012).

16. A. Gnauck, R. Tkach, A. Chraplyvy, and T. Li, "High-capacity optical transmission systems," J. Lightwave Technol. 26, 1032-1045 (2008).

17. B. Batsuren, H. H. Kim, C. Y. Eom, J. J. Choi, and J. S. Lee, "Optical VSB filtering of 12.5-GHz spaced $64 \times 12.4$ Gb/s WDM channels," J. Opt. Soc. Korea 17, 63-67 (2013).

18. G. B. Arfken and H. J. Weber, Mathematical Methods for Physicists, 6th ed. (Elsevier Academic, New York, USA, 2005).

19. C. Laperle and M. O'Sullivan, "Advances in high-speed DACs, ADCs, and DSP for optical coherent transceivers," J. Lightwave Technol. 32, 629-643 (2014). 